\title{
Entre la reforma y la disidencia. Experiencias de docentes formados para evaluar el desempeño desde la reflexión y la colectividad
}

\section{Between reform and dissent. Experiences of teachers trained to evaluate the performance since reflection and collaborated work}

Adriana Olvera López, Adrián Frausto Martín del Campo, Carlos Alberto Roque Gómez,
Eber Toledo Santos, José Alonso Vázquez Rudamas, Julio César Cruz Ángel
Nicacia Elidia González Escobar, Nicolás Valerio Girón y Yolibeth Salazar Cruz*

\section{RESUMEN}

El presente artículo es el resultado de sistematizar la experiencia de uso de un modelo de evaluación de desempeño docente basado en estándares, diseñado en México y probado en escuelas públicas mexicanas desde hace casi una década. Se inscribe en un momento en el que la evaluación del desempeño docente es tema de la agenda de política educativa y describe las implicaciones contextuales de la evaluación docente para la mejora; asimismo, analiza cada paso de la aplicación del modelo: registro de clase, valoración, conversación, elaboración de trayecto de mejora y seguimiento al trayecto. Los hallazgos muestran, entre otros, que es necesario tomar con seriedad que diversos actores escolares se involucren en la evaluación; que el modelo puesto en práctica desata un proceso de aprendizaje y coformación entre evaluadores; así como que, a partir de su evaluación, las y los docentes explicitan y amplían las expectativas sobre su quehacer, además que sitúan la mejora del mismo en los ámbitos individual y colectivo.

Palabras clave: evaluación, desempeño docente, estándares, educación básica

\begin{abstract}
This article is the result of systematizing the use of based on standards evaluation model of teacher's performance, designed in Mexico and tested in Mexican public schools for almost a decade. This work is published when evaluating teacher performance is an issue on the educational policy agenda. It describes contextual implications of a teacher's evaluation with improvement purposes; also analyzes each step of the model: class registration, evaluation, conversation, improvement path and monitoring. The findings show, among others, that it is necessary to take seriously that various school actors must get involved in teacher's assessment; the model implemented unleashes a learning process and co-training between evaluators; teachers can explicit and extend their expectations about their own work, in addition to improving it in individual and collective levels.
\end{abstract}

Key words: evaluation, teaching performance, standars, elementary and middle school

\footnotetext{
* Agradecimientos. Los autores de este texto participaron como docentes y alumnado en el seminario "Modelos de Evaluación con Estándares de Desempeño Docente", que forma parte de la maestría en Gestión y Asesoría Técnica a la Escuela, impartida en Chiapas por el Centro Regional de Formación Docente e Investigación Educativa. Esperamos que lo que a continuación se presenta contribuya al diálogo necesario por una evaluación del desempeño docente que sea pertinente. Lo que implica no solo llegar al aula, sino, y sobre todo, hacer allí lo que coadyuve efectivamente a la mejora. A casi diez años de su piloteo, la experiencia de estándares sigue vigente como ejemplo de que es posible reunir la reflexividad y el trabajo en colectivo, dos elementos esenciales en la labor del docente de educación básica.
} 


\section{ALGUNOS DATOS CONTEXTUALES}

En los últimos años, el sistema educativo mexicano ha transitado por una reestructuración complicada que inició en el ámbito legal. El gobierno federal apostó por una Reforma Educativa (RE) cuya premisa fue la de transformar el cuerpo normativo que sustenta el actuar del aparato, para propiciar los cambios necesarios en vías de mejorar la calidad educativa y aumentar la cobertura escolar.

Es importante recordar el clima político y social ${ }^{1}$ que rodeó la reforma al Artículo $3^{\circ}$ Constitucional: sin la oposición de la cúpula del sindicato de maestros (aunque sin el aval de las y los maestros, mucho menos de la disidencia magisterial), el gobierno federal llegó a un acuerdo con las tres principales fuerzas políticas del país para sacar adelante la reforma educativa y el resto de las llamadas reformas estructurales; sin embargo, la reforma al artículo ya mencionado se dio en un clima de tensión social, al grado que fue aprobada por el poder legislativo velozmente, sin discusión y en una sede distinta a la del Congreso de la Unión (Animal político, 2013).

Una vez modificada la Constitución, se llevó a cabo la adecuación de la Ley General de Educación (LGE) y la creación de la Ley General del Servicio Profesional Docente (LGSPD) (Diario Oficial de la Federación, 2013), donde se estableció que, para el ingreso, así como para la promoción en el servicio, las y los maestros deberían presentar un examen de oposición (Arts. 21 y 26), mientras que para la permanencia y el reconocimiento realizarían una evaluación de su desempeño (Arts. 52 y 45). El gobierno federal ha insistido en que la evaluación no es punitiva (siempre y cuando se participe en ella); no obstante, un sector del magisterio mantiene un clima de oposición a la reforma.

El modelo actual de evaluación de desempeño docente ha sido duramente cuestionado por dos razones: primero, ninguno de los instrumentos que se han utilizado hasta ahora recogía información directa de la práctica en el aula; y segundo, el gobierno no ha cumplido (hasta el momento de esta publicación) con el

${ }^{1}$ Véase La Jornada (11/12/2012, 26/02/2013, 22/08/2013); Animal Político (22/08/2013). 
pago de los incentivos que marca la ley de acuerdo con el resultado de la evaluación.

Un problema que se ha señalado acerca de la evaluación del desempeño es su correlato, a saber, la formación tanto inicial como continua. Por una parte, es necesario reformar los planes y programas de las escuelas Normales para formar a los próximos docentes en el marco del nuevo modelo educativo y propuesta curricular. Por otra, la propuesta de formación continua es incipiente, inadecuada y con poca oferta. Por si fuera poco, el sistema de acompańamiento a las y los maestros se ha rezagado pues, solo por mencionar algunos aspectos, aún no se ha implementado el sistema de tutorías a docentes noveles, y el Servicio de Asistencia Técnica a la Escuela (SATE) no termina por consolidarse. Esta fragilidad en el andamiaje de acompañamiento y formación docente no ha reclamado la atención mediática de forma tan intensa, como ha sucedido con las evaluaciones.

Es reduccionista creer que el magisterio solo se debate entre la aceptación irrestricta de la reforma y la disidencia de medidas extremas. Existen otros docentes cuyas experiencias se gestan de forma paralela a los tiempos de la reforma, pero con rumbos distintos a ella; en este sentido, son destacables las que no se sustentan en paradigmas de meritocracia, trabajo y esfuerzo individual, sino aquellas que apuestan al incentivo moral y a la mejora del quehacer docente a partir de su reflexión en colectivo.

Este artículo describe una de esas experiencias. En este caso, los protagonistas son un grupo de docentes del estado de Chiapas, estudiantes de un posgrado profesionalizante que les ha acercado a un modelo de evaluación de desempeño docente basado en estándares. En este artículo se ponen de relieve la puesta en práctica y los hallazgos que se derivan de ella; asimismo, se muestran las posibilidades de mejora generadas cuando la evaluación deja de ser un fin en sí mismo. Esta experiencia destaca también por haber sido realizada en el contexto mencionado, de gran disidencia.

Los estándares que las y los docentes emplearon forman parte del modelo Estándares para la Educación Básica en México, el cual fue diseñado para propiciar la mejora escolar mediante la aplicación de referentes a situaciones de evaluación donde participan diversos actores escolares. Este modelo se construyó, 
centralmente, a partir de la experiencia de escuelas, docentes y alumnado del sistema mexicano. Su primera versión fue sometida a procesos de legitimación y validación con la sociedad civil, autoridades y comunidades educativas. De todo ello existe un informe público (Centro de Estudios Educativos, 2008).

El uso del término "estándares" fue controversial, pues remitía a la idea de estandarización-homogeneización; preocupaba, sobre todo, que la evaluación invisibilizara las diferencias contextuales de cada escuela (cabe aclarar que esto no ha sido su propósito); no obstante, el modelo usó el término para denotar la existencia de referentes sobre los cuales situar el proceso de mejora escolar, referentes de los que carecía en ese momento el sistema educativo mexicano. Se optó por nombrarlos estándares o referentes, indistintamente.

Los Estándares para la Educación Básica en México abordan tres ámbitos de gran impacto en el aprendizaje: el currículo, el desempeño docente en el aula y la gestión escolar. El modelo cuenta con estándares específicos para cada ámbito y un proceso de aplicación común, que consiste en acercar a la escuela y sus actores los referentes con los cuales podrán valorar el aprendizaje del alumnado, la práctica docente y la gestión escolar; posterior a esa valoración, reflexionarán sobre sus capacidades y necesidades (fortalezas y debilidades), elaborarán trayectos de mejora y se comprometerán en su seguimiento.

Las y los docentes de Chiapas aplicaron solo los estándares de desempeño docente en el aula. Estos son unidades de información que aluden a una serie de acciones recurrentes y críticas que el cuerpo docente realiza durante su trabajo en el aula (Subsecretaría de Educación Básica, 2010: 23).

Estos estándares ayudan a que la evaluación de las prácticas sea útil para que las y los docentes:

- Emprendan un proceso de mejora continua de su desempeño.

- Reflexionen sobre su propia práctica docente o la de otros maestros.

- Evalúen e identifiquen fortalezas y debilidades en torno a aspectos específicos de la práctica.

- Identifiquen y organicen necesidades de formación (adiciona- 
les de los conocimientos disciplinares) tanto personales como colectivas (Subsecretaría de Educación Básica, 2010: 44).

El proceso de evaluación involucra a docentes, directivos y figuras de asistencia técnica a la escuela en una reflexión colectiva; son convocados a participar como autoevaluados (docentes), coevaluadores (pares del docente autoevaluado) y heteroevaluadores (directivos y figuras de asesoría técnica a la escuela).

El modelo cuenta con 25 estándares de desempeño docente en el aula, descritos, cada uno, en cuatro niveles de desempeño (Centro de Estudios Educativos, 2010a).

\begin{tabular}{|c|c|c|c|c|c|}
\hline & & Catego & as del desempeño doce & te en el aula & \\
\hline & Planeación & $\begin{array}{c}\text { Gestión del ambiente } \\
\text { de clase }\end{array}$ & Gestión Curricular & Gestión Didáctica & Evaluación \\
\hline & $\begin{array}{l}\text { Selección de } \\
\text { contenidos }\end{array}$ & $\begin{array}{l}\text { Relaciones } \\
\text { interpersonales }\end{array}$ & $\begin{array}{l}\text { Conocimiento de la } \\
\text { asignatura }\end{array}$ & $\begin{array}{l}\text { Presentación } \\
\text { curricular }\end{array}$ & Autovaloración \\
\hline & $\begin{array}{l}\text { Selección del } \\
\text { propósito }\end{array}$ & Manejo de grupo & $\begin{array}{l}\text { Relaciones entre } \\
\text { asignaturas }\end{array}$ & $\begin{array}{l}\text { Atención } \\
\text { diferenciada }\end{array}$ & $\begin{array}{l}\text { Valoración entre } \\
\text { alumnos }\end{array}$ \\
\hline & $\begin{array}{l}\text { Diseño de } \\
\text { estrategias } \\
\text { didácticas }\end{array}$ & & $\begin{array}{l}\text { Conexión asignaturas } \\
\text { contextos }\end{array}$ & $\begin{array}{l}\text { Organización del } \\
\text { grupo }\end{array}$ & $\begin{array}{l}\text { Valoración del } \\
\text { docente a los } \\
\text { alumnos }\end{array}$ \\
\hline 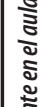 & $\begin{array}{l}\text { Selección de } \\
\text { mecanismos } \\
\text { de evaluación }\end{array}$ & & & $\begin{array}{l}\text { Relación de } \\
\text { aprendizaje } \\
\text { alumno-alumno }\end{array}$ & $\begin{array}{l}\text { Retroalimentación } \\
\text { de saberes }\end{array}$ \\
\hline ธ్ర & & & & $\begin{array}{l}\text { Recursos } \\
\text { didácticos }\end{array}$ & \\
\hline 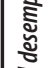 & & & & $\begin{array}{l}\text { Recursos } \\
\text { espaciales }\end{array}$ & \\
\hline ะั้ & & & & $\begin{array}{l}\text { Manejo del } \\
\text { tiempo }\end{array}$ & \\
\hline ֻँ & & & & Indicaciones & \\
\hline & & & & Explicaciones & \\
\hline & & & & Preguntas & \\
\hline & & & & $\begin{array}{l}\text { Actividades } \\
\text { dirigidas }\end{array}$ & \\
\hline & & & & $\begin{array}{l}\text { Actividades no } \\
\text { dirigidas }\end{array}$ & \\
\hline
\end{tabular}


En un cuadernillo especial (Centro de Estudios Educativos, 2010b) se ofrece a los participantes la descripción de cada estándar y pautas para observar cada referente. Un ejemplo es el que se muestra a continuación, y que pertenece al estándar Preguntas. Se puede notar que hay una casilla llamada "valoración", donde asignan el nivel de desempeño que más se acerca a lo observado en el aula; y "justificación”, donde cada evaluador ha de explicitar el porqué del nivel seleccionado.

\begin{tabular}{|c|c|c|c|c|c|}
\hline $\begin{array}{l}\text { Referentes } \\
\text { degestión } \\
\text { didáctica }\end{array}$ & $\begin{array}{c}\text { Estándares de gestión } \\
\text { didáctica }\end{array}$ & Pautas para observar & $\begin{array}{l}\text { Niveles de los estándares } \\
\text { de gestión didáctica }\end{array}$ & Valoración & Justificación \\
\hline \multirow{4}{*}{ Preguntas } & \multirow{4}{*}{$\begin{array}{l}\text { Durante la clase } \\
\text { formula, de manera } \\
\text { muy frecuente, } \\
\text { preguntas abiertas } \\
\text { que promueven } \\
\text { procesos de reflexión } \\
\text { sobre los saberes y } \\
\text { procedimientos de los } \\
\text { alumnos. }\end{array}$} & \multirow{4}{*}{$\begin{array}{l}\text { Durante la clase, } \\
\text { se observa si el } \\
\text { lenguaje usado por } \\
\text { el docente para hacer } \\
\text { preguntas propicia } \\
\text { o no la reflexión } \\
\text { sobre los saberes } \\
\text { (conocimientos) y } \\
\text { procedimientos de } \\
\text { los alumnos. Esto } \\
\text { se aprecia en el } \\
\text { tipo de respuestas } \\
\text { y explicaciones que } \\
\text { los alumnos dan } \\
\text { a las preguntas. } \\
\text { Si lo hacen con } \\
\text { respuestas reflexivas } \\
\text { y/o argumentativas } \\
\text { tienden a ser } \\
\text { abiertas, pero si son } \\
\text { memorísticas o de } \\
\text { "consentimiento" se } \\
\text { consideran cerradas. } \\
\text { En caso de que realice } \\
\text { preguntas abiertas, se } \\
\text { observa la frecuencia } \\
\text { con que lo hace a lo } \\
\text { largo de la clase. }\end{array}$} & $\begin{array}{l}\text { 1. Durante la clase } \\
\text { formula, preguntas } \\
\text { cerradas que no } \\
\text { promueven un } \\
\text { proceso de reflexión } \\
\text { sobre los saberes y } \\
\text { procedimientos de los } \\
\text { alumnos. }\end{array}$ & & \\
\hline & & & $\begin{array}{l}\text { 2. Durante la clase } \\
\text { formula, de } \\
\text { manera ocasional, } \\
\text { preguntas abiertas } \\
\text { que promueven } \\
\text { procesos de reflexión } \\
\text { sobre los saberes y } \\
\text { procedimientos de los } \\
\text { alumnos. }\end{array}$ & & \\
\hline & & & $\begin{array}{l}\text { 3. Durante la clase } \\
\text { formula, de } \\
\text { manera frecuente, } \\
\text { preguntas abiertas } \\
\text { que promueven } \\
\text { procesos de reflexión } \\
\text { sobre los saberes y } \\
\text { procedimientos de los } \\
\text { alumnos. }\end{array}$ & & \\
\hline & & & $\begin{array}{l}\text { 4. Durante la clase } \\
\text { formula, de manera } \\
\text { muy frecuente, } \\
\text { preguntas abiertas } \\
\text { que promueven } \\
\text { procesos de reflexión } \\
\text { sobre los saberes y } \\
\text { procedimientos de los } \\
\text { alumnos. }\end{array}$ & & \\
\hline
\end{tabular}


El uso de estándares sigue un proceso, que llevó a cabo el colectivo docente en Chiapas, previa capacitación, y consta de los siguientes pasos (Centro de Estudios Educativos, 2010a):

\section{Capacitación de los actores en el modelo}

Primero se da a conocer a docentes, directivos y miembros de la comunidad escolar el propósito de la evaluación, la forma de llevarla a cabo y lo que se puede obtener de ella. La capacitación ha resultado crucial, pues no solo se busca proveer la información del funcionamiento del modelo, sino también construir la confianza necesaria entre el equipo que va a vivir esta experiencia.

Hasta ahora se ha promovido un uso voluntario de los estándares de desempeño docente, lo que implica que el docente autoevaluado se proponga libremente, para que su práctica sea observada. Él o ella deciden la clase, tema, asignatura, grupo y duración de la misma; es decir, se puede preparar para ser evaluado. En esta fase también se nombra a quienes fungirán como coevaluador y heteroevaluador. Cada participante es capacitado con especificidad en cuanto a su rol.

\section{Registro de la práctica docente en una sesión de clase}

El registro se puede realizar en soporte de video, audio o papel (Centro de Estudios Educativos, 2010b), según las posibilidades, y la intención es que el docente pueda observarse después de clase y recuperar así elementos para su valoración. Coevaluador y heteroevaluador asisten a la clase y están atentos a los elementos que les servirán para valorar cada referente de práctica.

\section{Valoración de la práctica observada}

Cada evaluador realiza la valoración individualmente en un cuadernillo (Centro de Estudios Educativos, 2010b) que, como se dijo antes, contiene los 25 referentes de práctica. Se sugiere que, por cada referente, indique el nivel del desempeño (que va del 1 al 4) y justifique la selección de ese nivel. 


\section{Reflexión sobre la práctica valorada}

Las y los evaluadores se reúnen para reflexionar sobre la práctica docente. Acuden a conversar con los cuadernillos de valoración que cada uno ha completado. La sugerencia para este encuentro es que la conversación transcurra en un ambiente de confianza que posibilite a cada uno, y en particular al docente autoevaluado, la expresión de sus juicios argumentados sobre la práctica observada.

Este es uno de los pasos más importantes del proceso, pues se trata de sentarse a la mesa para comunicar desde lo observado (y no desde juicios o prejuicios), proponer y comprometerse con el cambio de la práctica del autoevaluado. Es un mecanismo que fortalece la comunicación y permite construir redes de apoyo al interior del centro escolar.

\section{Elaboración del trayecto de mejora}

La o el docente autoevaluado construye el trayecto de mejora de su práctica a partir de la conversación y reflexión con sus compañeros y autoridades. En ese trayecto se mencionan los aspectos de la práctica a atender, los recursos necesarios y los tiempos para lograrlo. Es posible que los evaluadores encuentren necesario el diseño de un trayecto de mejora para todo el colectivo docente y no solo para el autoevaluado, esto en función de los aspectos que hayan sido seleccionados y que se desee trabajar con varios docentes.

\section{Seguimiento al trayecto de mejora}

El docente pondrá en práctica el trayecto de mejora que ha diseñado, pero no estará solo en esta tarea, cuenta con el compromiso del coevaluador y heteroevaluador para darle seguimiento. Es recomendable que ese compromiso se manifieste por escrito en un plan que señale la periodicidad con la que visitarán al docente para conocer sus avances y lo que mostrará como evidencia del cumplimiento de su trayecto. En esas visitas se esperan conver- 
saciones que permitan al autoevaluado sentirse acompañado y retroalimentado.

Una vez alcanzadas las metas del trayecto, el proceso se puede emprender de nuevo, ya sea con el mismo docente u otros. La primera experiencia del centro escolar ha sentado las bases de un proceso dinámico, que tiene la forma de una espiral que avanza hasta incluir la evaluación de las prácticas de las y los docentes y la formación de cada integrante del centro escolar como evaluador.

Se ha mencionado que los docentes de Chiapas que han promovido este proceso en sus aulas, lo hicieron como parte del posgrado que cursan; pero antes que ellos, cientos de docentes emprendieron procesos como evaluadores de la práctica con los estándares.

La primera versión de los estándares se construyó de septiembre de 2007 a marzo de 2008, su legitimación y validación tuvo lugar de marzo a mayo de 2008, en octubre de ese mismo año se alistó la segunda versión que recogió los resultados de ambos procesos. Esa versión se piloteó en dos ocasiones. La primera, durante los ciclos escolares 2008-2009 y 2009-2010, cuando se realizaron tres levantamientos en una muestra de 608 escuelas (19 por entidad, todas ellas pertenecientes a las escuelas que pilotearon la Reforma Integral de la Educación Básica). La segunda, en los ciclos escolares 2009-2010 y 2010-2011, que llevó el modelo en escuelas de educación indígena, mediante los Asesores Académicos de la Diversidad (figuras de asesoría técnica a la escuela indígena), que fungieron como promotores de estándares. Estas dos experiencias permitieron que poco más de 900 escuelas iniciaran procesos de mejora en los ámbitos curricular, de desempeño docente en el aula y de gestión escolar.

En particular, los estándares de desempeño docente fueron utilizados para evaluar poco más de 1800 prácticas docentes, y se generaron más de 6000 valoraciones de esas prácticas (total de lo emitido por auto, co y heteroevaluadores) (Centro de Estudios Educativos, 2016. Presentación sobre estándares de desempeño docente en aula, documento interno).

Después de 2011, el modelo de estándares de desempeño docente en el aula se difundió mediante talleres en escuelas normales en el Estado de México y Nuevo León, y a miembros de 
asistencia técnica a escuelas en el estado de Hidalgo y la Ciudad de México.

Desde 2014 los estándares de desempeño docente en el aula han servido para diseñar parte de la oferta de posgrado del Centro Regional de Formación Docente e Investigación en Educación (CRESUR). El Centro de Estudios Educativos junto con CRESUR ha diseñado un diplomado en gestión de la evaluación, una maestría en evaluación educativa donde el proceso de evaluación mediante estándares es el organizador de los ejes curriculares; además de que la institución también ofrece un seminario de estándares dentro de la maestría en gestión educativa. El alumnado de este seminario fue quien aplicó el modelo.

\section{EXPERIENCIA DE LAS Y LOS DOCENTES EN CHIAPAS}

En los meses de mayo y junio de 2016, un grupo de siete docentes (dos mujeres y cinco hombres, uno de secundaria y seis de bachillerato), formaron parte del seminario "Modelos de Evaluación con Estándares de Desempeño Docente", que es una de las asignaturas de la maestría en Gestión y Asesoría Técnica a la Escuela, impartida en el CRESUR en la modalidad semipresencial.

El propósito del seminario era que el alumnado analizara y evaluara el desempeño docente a partir de la aplicación del modelo de evaluación con estándares. Se realizó una sesión presencial al inicio, en la que se capacitaron en cada una de las etapas del modelo; posteriormente, el trabajo a distancia implicó la puesta en práctica del modelo en el centro de trabajo de cada docente, o bien en otra escuela seleccionada por ellos mismos. El análisis siguiente se desprende de lo que llevaron a cabo en cada etapa.

\section{Presentación}

La primera actividad que realizaron las y los promotores de la evaluación con estándares fue invitar a las y los docentes interesados en participar. Días antes de que iniciaran este proceso había sido anunciado un paro magisterial. Ante la inminencia de la suspensión de labores, el profesorado promotor de la evaluación buscó alternativas para llevar adelante el procedimiento. 
La selección de las y los docentes a evaluar y ser evaluados fue de manera diversa por las condiciones coyunturales; algunos de los criterios fueron: por afinidad, por tomar el ejercicio con seriedad, compañerismo, perfil, empatía y hubo, incluso, quien seleccionó a docentes que tuvieran poca experiencia frente a grupo o que impartieran materias con altos índices de reprobación como Matemáticas.

El paso que siguió a la invitación fue presentar la finalidad y pasos de la evaluación con estándares de desempeńo. Para ello organizaron en cada escuela una reunión informativa con las y los docentes que habrían de intervenir en la evaluación, con el fin de explicar el procedimiento, compartir los instrumentos y acordar las fechas en que se realizaría el registro de clase, la valoración, trayecto de mejora y procedimiento.

\section{Registro de clase}

A continuación se presenta una tabla donde se muestran las materias, la temática de la clase observada, el semestre y la duración de la clase registrada (todas por videograbación). Todos y todas las maestras que fueron observadas en el proceso de evaluación fueron de nivel preparatoria. Hubo dos de matemáticas, dos de Taller de lectura, dos de Agricultura y una de Física. De la información con la que se cuenta, tres asignaturas fueron de segundo semestre, dos de cuarto, uno de último semestre; no se contó con información de una asignatura al respecto. La duración de la clase también varió en un rango de 17 a 50 minutos.

\begin{tabular}{|l|l|c|c|c|}
\hline \multicolumn{1}{|c|}{ Asignatura } & \multicolumn{1}{|c|}{ Tema } & Nivel educativo & Semestre & Duración \\
\hline Física & Circuito & Preparatoria & 6 to & ND \\
\hline Matemáticas & ND & Preparatoria & 2 do & 17 minutos \\
\hline Matemáticas & Funciones polinómicas & Preparatoria & 4 to & 29 minutos \\
\hline Taller de Lectura & Época medieval & Preparatoria & 4 to & ND \\
\hline Taller de Lectura & El cuento & Preparatoria & ND & 50 minutos \\
\hline Agricultura & Sistemas de producción agrícola & Preparatoria & $2 \mathrm{do}$ & 40 minutos \\
\hline Agricultura & Sistemas de producción & Preparatoria & $2 \mathrm{do}$ & 50 minutos \\
\hline
\end{tabular}


Un elemento clave en el proceso de selección de docentes fue la confianza, ya que no resulta sencillo generar un espacio de evaluación donde el asunto a evaluar sea la práctica profesional. Incluso, un promotor identificó como oportunidad de mejora el clima favorable para generar un espacio franco y respetuoso de intercambio de valoraciones.

Quienes participaron en la evaluación de las diferentes escuelas fueron docentes y personal directivo de las mismas escuelas; no hubo participantes externos. Esto es importante porque el diálogo se dio entre el personal que se acompaña cotidianamente en el quehacer educativo, aumentando la posibilidad de fortalecer la evaluación y el aprendizaje colegiado.

Las y los promotores señalaron que la principal dificultad con la que se enfrentaron las personas evaluadas fue la de ser observados y videograbados; sin embargo, coincidieron también en la importancia de la conversación previa donde se les presentó el objetivo y el procedimiento de la evaluación, lo consideraron un buen espacio para aclarar dudas y brindar confianza a las y los docentes que habrían de ser evaluados. Los principales aprendizajes que reportaron las y los promotores del registro de la clase fueron:

- Evaluar a sus pares les da la oportunidad de conocer la manera en que otros docentes de su mismo nivel educativo planean y desarrollan una clase. Valoraron la posibilidad de aprender de las estrategias pedagógicas del profesorado cuya práctica está observándose.

- También fue una oportunidad para conocer la práctica docente de otros niveles educativos. Para un promotor de la evaluación que pertenece al nivel secundaria, resultó novedoso evaluar a un docente de nivel medio superior, y así conocer las similitudes y diferencias respecto a su nivel, y la interacción entre los colegas de nivel medio.

- Las y los promotores reconocieron la importancia de la correcta aplicación de los instrumentos, porque estos les permitieron observar con atención lo que sucedía en el aula, y posteriormente plantear sugerencias pertinentes a sus pares evaluados. 


\section{Valoración}

Para arribar a la valoración es necesario haber presenciado la clase y hecho el contraste entre lo observado y los referentes de desempeño. Esto incluye no solo asignar el nivel a cada una de las 25 acciones del aula, sino también justificar la selección del mismo. El alumnado del seminario compartió solo las valoraciones, que fueron sistematizadas de la siguiente manera:

a) Valoración global: promedio de los valores asignados a los 25 referentes.

b) Valoración por categorías: promedio de los valores asignados a los referentes que integran cada categoría (planeación, gestión del ambiente de clase, gestión curricular, gestión didáctica y evaluación).

c) Valoración por referentes: promedio de los valores asignados a cada uno de los 25 estándares.

d) Valoración por evaluador:

1. Autoevaluado: promedio de las valoraciones emitidas por las y los docentes que evaluaron su práctica.

2. Coevaluador: promedio de las valoraciones emitidas por los docentes que acompañaron a su par en la evaluación de su práctica.

3. Heteroevaluador: promedio de las valoraciones emitidas por la autoridad escolar o de zona que acompañó al docente en la evaluación de su práctica.

Las valoraciones a cada referente pueden ser del 1 al 4; para hacer más comprensible esta escala, los resultados que aquí se presentan se transformaron a una escala de 25 a 100.

En total se evaluaron siete prácticas docentes y el número de valoraciones (incluyendo auto, co y heteroevaluación) fue de 18, distribuidas de la siguiente manera:

- Cinco prácticas contaron con los tres evaluadores previstos por el modelo.

- Una contó con dos evaluadores (auto y co).

- De una práctica solo se conoció la coevaluación. 
En la siguiente gráfica se muestran las valoraciones globales para cada práctica docente que contó con más de un evaluador. Dichas valoraciones oscilaron de 46.3 a 84.7. La mayor valoración es indicador de que las prácticas se apegan a lo que señalan los referentes. En este caso llama la atención la gran distancia que existe entre la práctica menos valorada y las demás evaluadas.

GRÁFICA 1. Valoraciones globales de cada docente evaluado

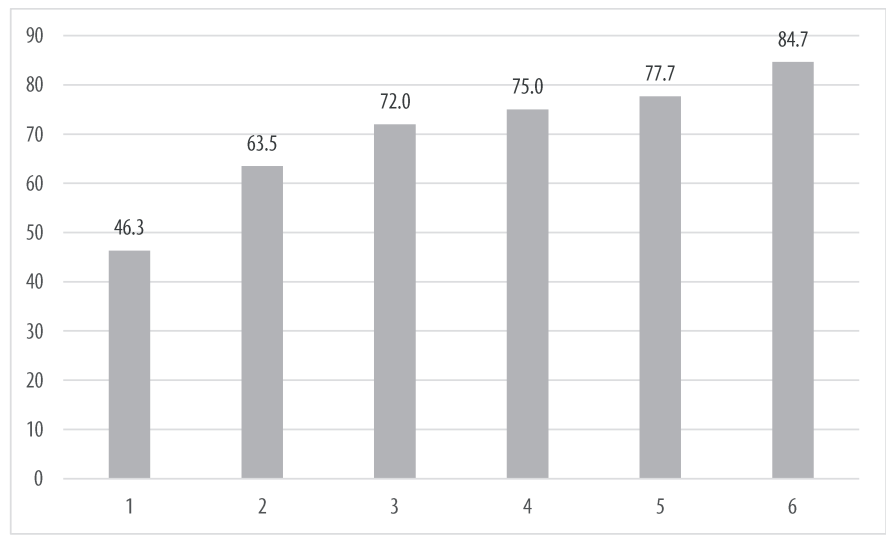

En cuanto a los resultados por categoría, derivados del promedio de todas las valoraciones, se tiene que la mejor valorada es gestión del ambiente de clase, seguida de la planeación y gestión curricular. Gestión didáctica y evaluación son las que menor puntaje tienen. Llama la atención el caso de la planeación, que tiene como valor mínimo un puntaje menor que la gestión didáctica.

\section{GRÁFICA 2. Resultados por categorías}

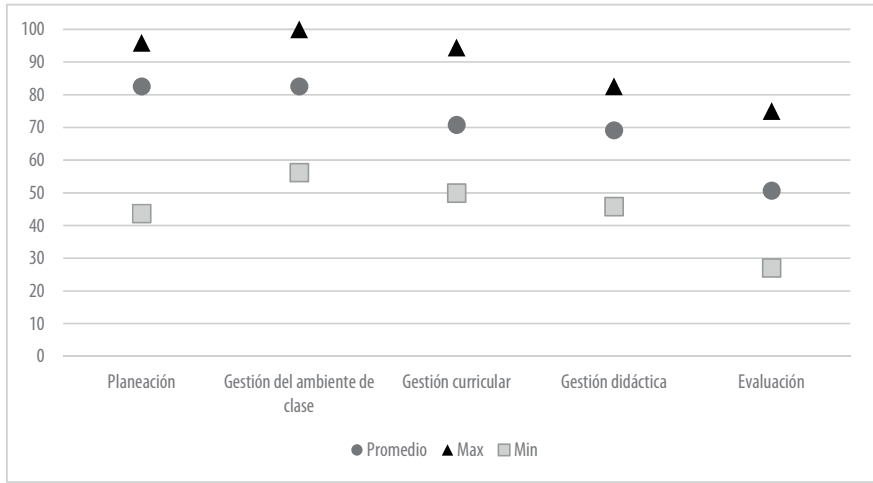


Por lo que respecta a los referentes, aquellos con menor puntaje son la valoración entre alumnos y actividades no dirigidas. Esto indica que ambos aspectos todavía no son promovidos de forma importante al interior de las aulas. Selección de contenidos, conocimiento de la asignatura y explicaciones fueron los tres mejor valorados.

GRÁFICA 3. Valoraciones por referente

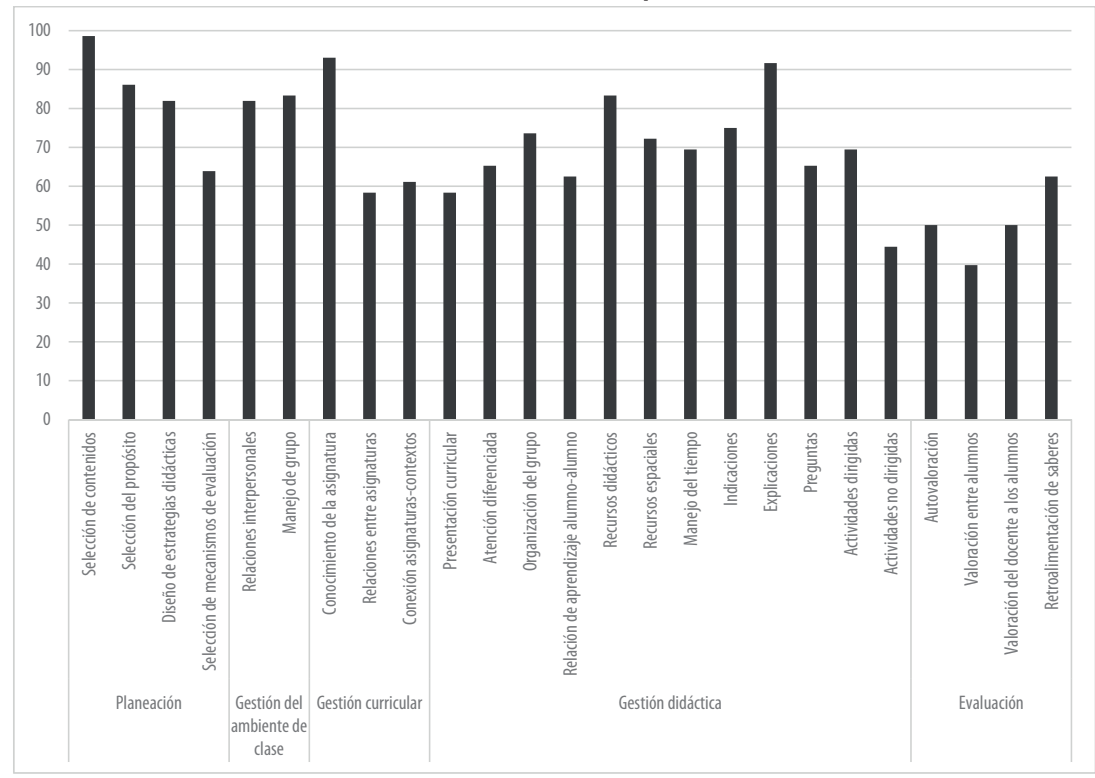

En cuanto a las valoraciones por tipo de evaluador, se encontró que los puntajes que asignaron quienes fungieron como autoevaluados son muy cercanos a lo que ya se reportó en las valoraciones promedio por referente. Ellas y ellos son particularmente críticos con la categoría evaluación y consideran que su fortaleza es la selección de contenidos, conocimiento de la asignatura, así como las explicaciones.

Las y los coevaluadores a su vez, señalan que las áreas de mejora se encuentran en la valoración entre alumnos y las actividades no dirigidas; aprecian que las fortalezas son la selección de contenidos y del propósito, así como las explicaciones (en esto coinciden con las y los autoevaluados). Asimismo, son críticos con la gestión cu- 
rricular que desempeñan sus compañeros, aunque rescatan el conocimiento de la asignatura que mostró el o la docente.

En cuanto a las y los heteroevaluadores, coinciden también en que las prácticas pueden mejorar en cómo se realiza la valoración entre alumnos y las actividades no dirigidas; en cuanto a las fortalezas, las ubican en la selección de contenidos, conocimiento de la asignatura y recursos didácticos. En general asignan mayor valoración por referente, en comparación con auto y coevaluadores.

Las y los autoevaluados valoran mejor su práctica que co y heteroevaluadores en solo ocho de los 25 referentes: presentación curricular, organización del grupo, relación de aprendizaje alumno-alumno, recursos espaciales, manejo del tiempo, indicaciones, actividades dirigidas y retroalimentación de saberes.

Las y los coevaluadores valoran mejor a sus compañeros en siete referentes: selección del propósito, atención diferenciada, explicaciones, preguntas, actividades no dirigidas, valoración entre alumnos, valoración del docente a los alumnos.

Por su parte, las y los heteroevaluadores valoran mejor nueve referentes: diseño de estrategias didácticas, selección de mecanismos de evaluación, relaciones interpersonales, manejo de grupo, conocimiento de la asignatura, relación entre asignaturas, conexión asignaturas-contextos, recursos didácticos y autovaloración.

GRÁFICA 4. Valoraciones por referente según tipo de evaluador

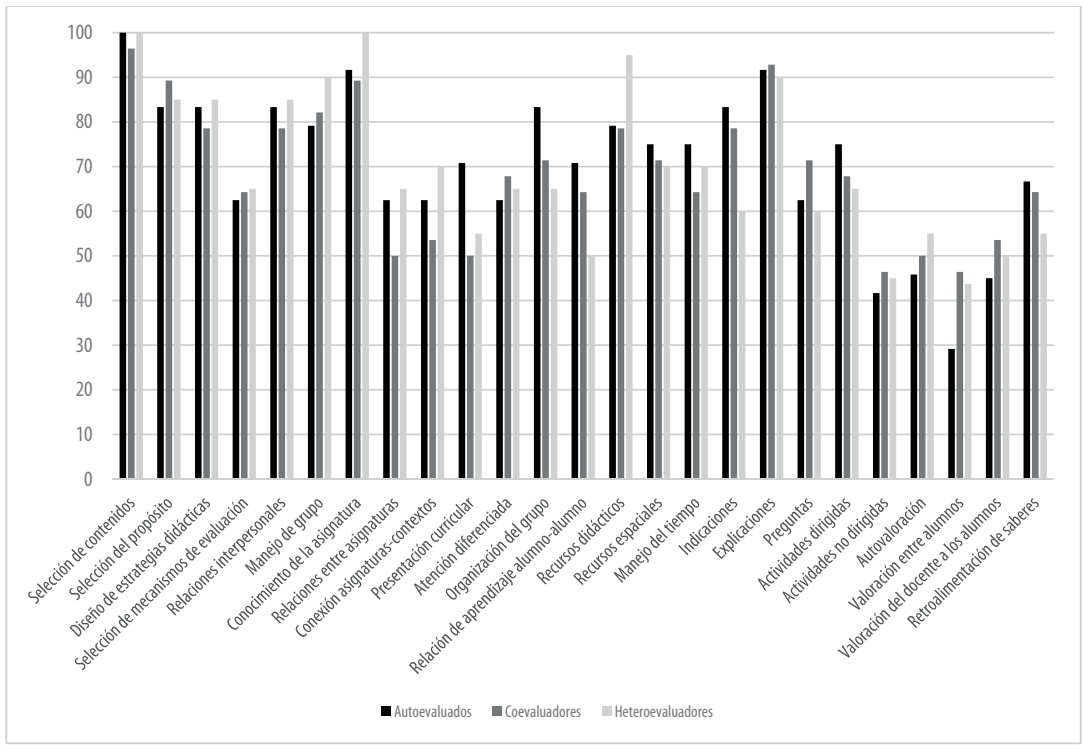


ENTRE LA REFORMA Y LA DISIDENCIA. EXPERIENCIA DE DOCENTES FORMADOS...

\section{CONVERSACIÓN}

La conversación es el momento en el proceso de la evaluación en que autoevaluado, coevaluador y heteoevaluador dialogan en torno a la práctica docente observada. Esta reunión es importante porque pretende promover el intercambio de opiniones, valoraciones y experiencias entre los participantes -a partir de la clase observada y registrada-, para identificar las fortalezas y las debilidades, tanto individuales como colectivas sobre la práctica docente con el fin de enriquecerla, modificarla o ajustarla, a partir de un trayecto de mejora y un acompańamiento pertinente.

Las y los promotores de la evaluación reportaron que en la conversación estuvieron presentes el auto, el co y el heteroevaluador, principalmente. En dos casos se contó con asistentes extras que se interesaron en observar el diálogo entre el colectivo evaluador. La manera en que se condujo la conversación fue diferente: hubo quien mencionó que, gracias al clima de confianza, no hubo necesidad de establecer roles o reglas para conversar; en cambio, hubo un promotor que estableció ciertas condiciones de diálogo como invitar a las y los participantes a enfocarse en la información registrada en los instrumentos de valoración, y llevar un registro de las observaciones y comentarios de los otros evaluadores.

Lo reportado por las y los promotores destaca que, en su mayoría, el formato de procedimiento sobre la conversación fue semiestructurada, esto es: se retomaron las fortalezas y debilidades, y tomaron en cuenta los indicios para conversar y poner en común los hallazgos a partir de las valoraciones. Además el clima en el que se dio el intercambio de valoraciones y justificaciones fue de confianza y respeto mutuo. Fue importante también la apertura por parte de las y los docentes evaluados para recibir comentarios sobre su práctica.

En general esta etapa del proceso de evaluación fue valorada positivamente. El clima de respeto y confianza construido entre las y los promotores de la evaluación con el personal docente participante en este proceso, además de la claridad con la que se intercambiaron las valoraciones en torno a la práctica docente, permitió la construcción de consensos respecto a las oportunida- 
des de mejora de las y los docentes evaluados. También señalaron lo siguiente:

- La claridad en la comunicación entre evaluadores se debió, en buena medida, a que la conversación estuvo centrada en los referentes y en las categorías de desempeño en el aula.

- Las y los evaluadores expusieron la justificación de sus valoraciones. La comparación de estas permitió llegar a consensos sobre el desempeño y sobre por dónde de vislumbraría una ruta de mejora de la práctica.

- Se suscitaron contrastes interesantes en el intercambio de valoraciones, como el que un autoevaluado se percatara de que su desempeño no está por debajo de lo que consideraba, lo cual le permitió hacer una síntesis entre su percepción y la mirada externa.

- En general, reconocieron el beneficio de que la práctica sea observada por otros y por ellos mismos, en función de caracterizar las fortalezas y las debilidades en perspectiva de la mejora continua.

Las y los docentes manifestaron que la conversación se convirtió en un espacio que, además de intercambiar los valores de cada estándar con el fin de detectar las fortalezas y oportunidades de cambio, permitió compartir estrategias pedagógicas y vislumbrar posibles campos de formación a futuro. Esto es importante porque destaca el carácter formativo de la evaluación.

\section{TRAYECTOS DE MEJORA}

Los trayectos de mejora concretan la reflexión y establecen las condiciones para transformar la práctica. Las y los promotores de la evaluación reportaron la elaboración de seis trayectos; es decir, se desconoce el trayecto de mejora de una de ellas. En su estructura, los trayectos incluyeron diversos apartados; el análisis realizado consistió en ubicar los rasgos semejantes de cada uno y clasificarlos en aspectos generales, propósitos, acciones, recursos, tiempos y resultados. 


\section{Aspectos generales}

Tres de los que emprendieron el ejercicio consideraron las categorías como punto de partida para plantear los trayectos de mejora. Uno definió como aspectos generales, los antecedentes del trabajo con estándares (el que se deriva de un proceso de análisis y reflexión y la metodología usada).

Uno más especificó referentes sobre los que trabajaría el docente.

Gestión curricular. No se observa la relación entre asignaturas.

Y uno señaló las estrategias que habrían de emprender, sin hacer explícito alguna categoría o referente.

Estructurar diversas estrategias que ayuden a mejorar la enseńanza en cada uno de los alumnos.

\section{Propósitos}

Los propósitos estuvieron asociados a los aspectos generales descritos. En función de qué tan generales o particulares fueron esos aspectos es que los propósitos arribaron a descripciones más o menos cercanas al quehacer en el aula.

Sobre todo refirieron al plan de clase, interacción de alumnos, contenidos de asignatura y evaluación.

Por ejemplo, lo mencionado respecto a la interacción y participación de alumnos:

- Básicamente se persigue la interacción entre los alumnos y la expresión de sus ideas.

- Crear actividades para que los alumnos interactúen más.

- Corregir la práctica docente dentro del aula con la ayuda de las diversas estrategias didácticas que mejoren la participación de los alumnos dentro de la misma.

- Que los estudiantes interactúen entre ellos en las diferentes actividades para fortalecer el vínculo afectivo, además de compartir sus opiniones. 
En uno de los trayectos se encuentra que la redacción de propósitos es en primera persona, lo que alude más cercanamente al compromiso hecho por el docente:

Ser un mejor docente y que a todos mis alumnos les guste mi asignatura y que aprendan, que entiendan, que vean más allá.

\section{Acciones}

Las acciones son la explicitación de tareas que realizará el docente para cumplir sus propósitos. Las mencionadas en los trayectos se pueden agrupar en cinco temáticas:

- Estrategias didácticas: rediseño de estrategias, revisión y diseño de materiales didácticos, promover participación de alumnos, trabajo colaborativo, incorporar herramientas digitales, permitir que los alumnos se organicen por afinidad, prácticas de campo.

- Formación de los docentes: talleres, cursos de capacitación, preparación continua (leer, escribir, buscar materiales de su asignatura, indagar).

- Trabajo colegiado: reuniones por línea curricular, planeación, proyectos integradores, que cada uno desarrolle su trayecto de mejora.

- Planeación: introducir mejoras, adecuar a los propósitos de la asignatura, contextualizarla.

- Procesos educativos: seguimiento, autovaloración, métodos de evaluación por unidades, evaluación de acuerdo con el tema y con las actividades.

- Portafolio docente: promover su implementación.

Estas acciones son la síntesis de lo que los evaluadores consideran como su campo de acción, tanto a nivel personal como colectivo. Algunas de ellas denotan una influencia muy fuerte de los estándares, por ejemplo: el rediseño de estrategias didácticas y la autovaloración; otras son los prerrequisitos de la transformación: talleres, formación en general; y algunas más tratan sobre asuntos que forman parte de su quehacer cotidiano, pero que 
pueden innovar, como en el caso de contextualizar la planeación o el desarrollar proyectos integradores en la escuela.

\section{Recursos}

La gran mayoría de los recursos enlistados está en relación con lo que los docentes podrán ocupar en el aula, y se pueden distinguir: programas de asignatura, mobiliario y equipo tecnológico (computadora, cañón, lap top), material didáctico (crayolas, papel bond, postes, madera, agua, semillas e implementos agrícolas), listas de cotejo, rúbricas y formatos diversos destinados a la evaluación.

Sólo en un caso se hace alusión a la necesidad de contar con CRESUR para capacitar con mayor profundidad a los docentes, así como buscar apoyo en la Secretaría de Educación Pública, mediante el asesor técnico pedagógico o cursos en línea.

\section{Tiempo de realización}

Quienes mencionan la temporalidad de sus acciones, refieren con mayor frecuencia que han de realizarlas continuamente o durante la clase. Otras periodicidades importantes son: cada que se realice la planeación y semanalmente.

\section{Resultados esperados}

Los resultados que se identificaron en los trayectos de mejora se pueden agrupar en cinco categorías:

- Docentes: docentes capaces de analizar y evaluar su desempeño, fortalecimiento de la interacción del colectivo docente, mejorar la práctica en beneficio de los alumnos, continuar creciendo profesionalmente, satisfacción personal, fortalecimiento de la práctica.

- Estrategias didácticas: implementación de estrategias de enseñanza y aprendizaje innovadoras, aprendizaje de las asignaturas como experiencia gratificante, relación de los contenidos con los contextos, claridad de los avances de los estudiantes 
respecto a los aprendizajes esperados, mejorar estrategias para un mejor aprendizaje.

- Planeación: adecuada, clara, precisa, bien dosificada, con propósitos y objetivos claros.

- Gestión del ambiente de clase: interacción de estudiantes en un ambiente armónico, agradable.

- Alumnos: mejores resultados académicos, aprendizajes significativos, logro de objetivos concretos, jóvenes que se motivan a seguir estudiando, terminan bachillerato, aplican los contenidos a la resolución de problemas cotidianos, aceptación y gusto de la materia.

\section{Seguimientos}

Los seguimientos son documentos donde se manifiestan los compromisos hechos por los evaluadores para acompańar al docente autoevaluado en la puesta en marcha de su trayecto. En este caso, al igual que sucedió con el análisis de los trayectos, se consideraron los elementos de semejanza en estructura para organizar y sintetizar los contenidos. Se identificaron las siguientes secciones: acciones a emprender, responsables, tiempos y recursos.

\section{Acciones a emprender}

Las acciones enunciadas en los seguimientos se encuentran estrechamente relacionadas con los elementos a trabajar en el trayecto de mejora. Además se incluyen otras acciones que son de índole escolar y, otras muy particulares sobre cómo continuar con el proceso mismo de evaluación del desempeño.

- Estrategias didácticas: diseño de estrategias, rediseño de materiales, trabajo en equipo, actividades fuera del salón de clase, actividades lúdicas y material de apoyo audiovisual.

- Trabajo en colectivo: reuniones de academia, reuniones de colectivo para plan de trabajo escolar, proyectos integradores, eventos que impliquen la interacción de todos los docentes de todas las asignaturas y de todos los alumnos de todos los grados, planeación didáctica argumentada, reuniones colegiadas.

- Portafolio docente: elaborarlo e implementarlo. 
- Planeación: revisar las concepciones teóricas, el cumplimiento de los propósitos en cada unidad, adecuar los contenidos necesarios, revisar propósitos específicos de la asignatura, establecer propósitos de acuerdo con las competencias, establecer criterios de evaluación al iniciar el tema.

- Proceso de mejora mediante estándares: evaluación del proceso, saber si el docente trabaja de forma individual o colectiva, avances y dificultades en el trayecto, replantear o adecuar el trayecto de mejora, implementar el trayecto de mejora, evaluación diagnóstica al inicio del semestre.

- Evaluación de alumnado: actividades que permitan la evaluación formativa y constante del alumnado, instrumentos que permitan una valoración más objetiva del desempeño de las y los estudiantes.

- Otra: analizar el perfil de egreso de los estudiantes.

\section{Responsables}

De 38 acciones a dar seguimiento, 18 son la responsabilidad de docentes de asignatura, 11 de directores y subdirectores de escuela. El resto se reparte entre el colectivo docente, las academias y otras figuras, como el secretario académico y los presidentes de academia.

\section{Tiempos}

La periodicidad para desarrollar cada actividad es muy variada, en general, se opta por dar seguimiento de forma continua durante el semestre; es igualmente común que quede abierto realizarlo cuando sea necesario.

Se presentan menciones a periodos de cuatro meses y semestral. También se hace referencia a que se realizaría al final de cada módulo o unidad, y en algunos casos durante la planeación, justo antes que inicie el semestre.

\section{Recursos}

Estos son casi idénticos a los planteados en el trayecto de mejora, es decir, incluyen los materiales, humanos y de infraestructura. 
Destaca que se hace alusión, de manera particular, a la necesidad de contar con espacios para las reuniones.

\section{Coincidencias entre valoraciones, trayectos de mejora y seguimientos}

El modelo de estándares apuesta a que los referentes contribuyen, vía la reflexión, a transformar la práctica; se reconoce que no se trata de una relación lineal, pues esa transformación se ve influida por múltiples factores adicionales a los estándares: la formación del maestro, sus necesidades particulares, las que considera más urgentes, el contexto inmediato, las expectativas personales, etc.; pero su virtud radica en que pone al alcance elementos clave de su práctica, que pueden ser determinantes en los aprendizajes de sus alumnos.

Partiendo de esta premisa, se realizó el ejercicio de coincidencias entre las valoraciones a cada referente y los contenidos temáticos de los trayectos de mejora y seguimiento.

El resumen de ese ejercicio se encuentra en la siguiente tabla, que presenta los seis casos de prácticas docentes que cuentan con valoraciones, trayecto de mejora y seguimiento. En la línea "valoración" pueden leerse los promedios de auto, co y heteroevaluador (en escala 25 a 100), para cada uno de los 25 referentes; las celdas que tienen color son las de los referentes con una valoración igual o menor a 50. En las filas "trayecto de mejora" y "seguimiento", se señala con una X la asociación de contenidos del trayecto y seguimiento con los referentes.

Caso 1. Las acciones del trayecto de mejora y del seguimiento se centran en la categoría planeación, vista como el medio para modificar lo que tiene lugar en el aula. En la fila de valoración esta categoría aparece con altos puntajes. Ninguno de los referentes menos valorados es atendido por el trayecto y el seguimiento.

Caso 2. Se aprecia que el trayecto de mejora se relaciona con tres de los referentes más bajos (relaciones entre asignaturas, relaciones interpersonales y presentación curricular), y a uno de puntaje elevado (selección de mecanismos de evaluación). En el seguimiento se observan acciones que ayudarán a que este último referente pueda llevarse adecuadamente al aula, mientras que el 
resto queda desatendido. Y se decide además, dar seguimiento a un aspecto no contenido en el trayecto (organización del grupo).

Caso 3. Los contenidos se asocian a los mismos referentes que en el caso 2, pero aquí, solo dos de los incluidos en el trayecto son de baja valoración (selección de mecanismos de evaluación y relaciones entre asignaturas), y se consideran dos de valoración más alta (presentación curricular y relaciones interpersonales). Selección de mecanismos de evaluación y organización del grupo (que no está contenido en el trayecto) son a los que se les dará seguimiento. Cabe mencionar que caso 2 y caso 3 comparten como heteroevaluador a la misma figura escolar, lo que estaría mostrando que, pese a que se trata de trayectos y seguimientos individuales, es probable que se haya llegado a acuerdos para avanzar en colectivo.

Caso 4. El trayecto versa temáticamente sobre cinco referentes, dos de ellos de baja puntuación (valoración entre alumnos y valoración del docente a los alumnos), y tres de puntuación media (presentación curricular, retroalimentación de saberes y autovaloración). En el seguimiento, se atienden cuatro de los referentes mencionados; solo se excluye la presentación curricular.

Caso 5. El trayecto de mejora atiende hasta ocho referentes; solo dos de ellos se encuentran entre los de más bajos puntajes de esta práctica (atención diferenciada e indicaciones); puntajes que serían medios para los otros casos. El seguimiento no hace alusión a tareas específicas por referente.

Caso 6. En este caso, solo se incluye en el trayecto una temática relacionada con el diseño de estrategias didácticas, que está entre los referentes mejor valorados. El seguimiento contempla otros temas no asociados a referentes o categorías de estándares.

De este ejercicio surgen diversas interrogantes que dan pie a futuras investigaciones sobre la mejora de la práctica: ¡cómo se toma la decisión de qué atender respecto de la práctica observada?, ¿cómo priorizan las acciones y temáticas los evaluadores? 


\begin{tabular}{|c|c|c|c|c|c|c|c|c|c|c|c|c|c|c|c|c|c|c|}
\hline 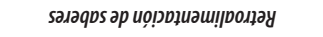 & $\stackrel{n}{n}$ & & & $\stackrel{n}{n}$ & & & $\infty$ & & & 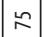 & $\times$ & $\times$ & 2 & $\times$ & & $m$ & & \\
\hline 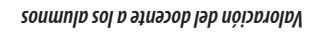 & $\infty$ & & & 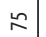 & & & $\Re$ & & & 2 & $\times$ & $x$ & 2 & $\times$ & & $\tilde{m}$ & & \\
\hline 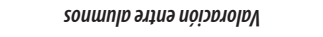 & $\infty$ & & & 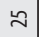 & & & 요 & & & 2 & $\times$ & $\times$ & $\stackrel{n}{\sim}$ & $\times$ & & 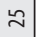 & & \\
\hline 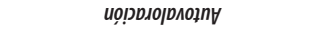 & $\approx$ & & & 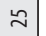 & & & 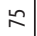 & & & $\infty$ & $\times$ & $\times$ & 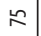 & $\times$ & & $m$ & & \\
\hline sвp!6!!! & $\approx$ & & & 유 & & & $\dddot{f}$ & & & 요 & & & 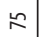 & $\times$ & & $\Re$ & & \\
\hline 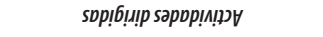 & $\tilde{6}$ & & & 유 & & & n & & & $\varnothing$ & & & $\kappa$ & $\times$ & & $\stackrel{n}{n}$ & & \\
\hline splunbадd & 으 & & & $\stackrel{n}{n}$ & & & $\kappa$ & & & 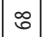 & & & 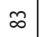 & & & $\infty$ & & \\
\hline 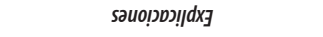 & $\kappa$ & & & $\stackrel{n}{n}$ & & & 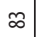 & & & $n$ & & & 8 & & & ○ & & \\
\hline 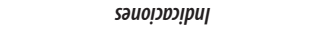 & $\infty$ & & & $\stackrel{n}{n}$ & & & n & & & $n$ & & & $\infty$ & $\times$ & & $\infty$ & & \\
\hline 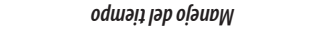 & $\infty$ & & & $\stackrel{n}{n}$ & & & $\approx$ & & & $\approx$ & & & $\approx$ & & & 요 & & \\
\hline sәр!̣गdsa sosin)วy & $\stackrel{n}{\sim}$ & & & 유 & & & ○ & & & $\infty$ & & & $\infty$ & & & ○ & & \\
\hline 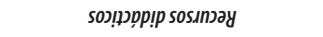 & 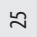 & & & 유 & & & 으 & & & 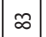 & & & $\approx$ & & & ○ & & \\
\hline 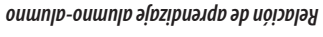 & 유 & & & $\stackrel{n}{\sim}$ & & & 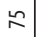 & & & 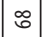 & & & $\stackrel{n}{n}$ & & & $\stackrel{n}{n}$ & & \\
\hline 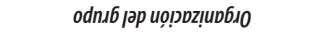 & $\tilde{6}$ & & & $\stackrel{n}{n}$ & & $\times$ & $\infty$ & & $\times$ & $\infty$ & & & 2 & & & 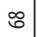 & & \\
\hline 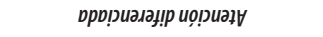 & $\stackrel{n}{n}$ & & & 유 & & & 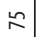 & & & $\infty$ & & & $\infty$ & $\times$ & & 은 & & \\
\hline 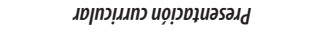 & 유 & & & 유 & $\times$ & & $\tilde{\infty}$ & $\times$ & & $\infty$ & $\times$ & & 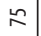 & & & $\cong$ & & \\
\hline 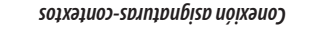 & $\approx$ & & & 유 & & & 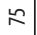 & & & $\approx$ & & & $\approx$ & & & $\infty$ & & \\
\hline 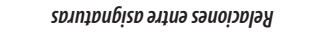 & $\approx$ & & & $\approx$ & $\times$ & & $\infty$ & $\times$ & & n & & & 8 & & & 요 & & \\
\hline 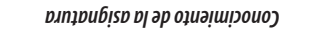 & 으 & & & $\stackrel{n}{n}$ & & & 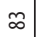 & & & $\approx$ & & & 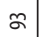 & & & 은 & & \\
\hline odnג6 әр о!วuрW & 유 & & & 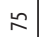 & & & 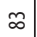 & & & 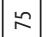 & & & 8 & & & $\infty$ & & \\
\hline 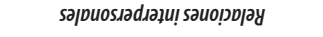 & 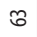 & & & 유 & $\times$ & & 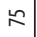 & $\times$ & & $\approx$ & & & 음 & & & ○ & & \\
\hline 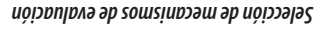 & $\stackrel{n}{x}$ & $\times$ & & $\stackrel{n}{n}$ & $\times$ & $\times$ & $\approx$ & $\times$ & $\times$ & $\infty$ & & & 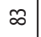 & $x$ & & $\approx$ & & \\
\hline 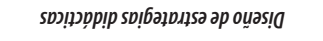 & 음 & $\times$ & $\times$ & $\kappa$ & & & ○ & & & $\approx$ & & & 8 & & & $\approx$ & $\times$ & \\
\hline 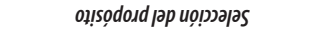 & 응 & $\times$ & & 응 & & & ○ & & & $n$ & & & 응 & & & ○ & & \\
\hline \multirow[t]{3}{*}{ 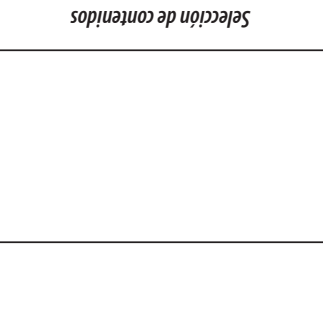 } & 음 & $\times$ & $\times$ & 응 & & & 음 & & & 은 & & & ○ & & & ๓ & & \\
\hline & 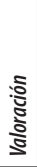 & 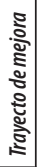 & 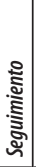 & 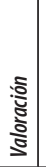 & 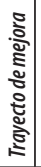 & 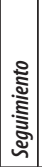 & 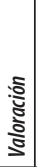 & 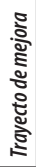 & 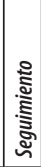 & 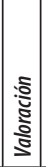 & 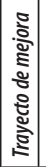 & 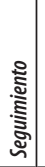 & 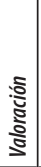 & 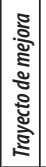 & 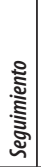 & 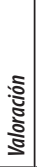 & 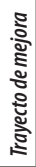 & 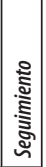 \\
\hline & & స్心 & & & 芯 & & & 芯 & & & $\begin{array}{l}\text { 巳 } \\
\stackrel{0}{0}\end{array}$ & & & $\begin{array}{l}\tilde{O} \\
\stackrel{\Xi}{\sigma}\end{array}$ & & & 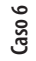 & \\
\hline
\end{tabular}


ENTRE LA REFORMA Y LA DISIDENCIA. EXPERIENCIA DE DOCENTES FORMADOS...

\section{CONCLUSIONES}

La sistematización de la experiencia del seminario "Modelos de Evaluación con Estándares de Desempeño Docente" ha permitido acercarse a una evaluación donde los protagonistas son actores escolares. Las relaciones laborales y personales que ellos viven median la manera en que un proceso de esta naturaleza puede llevarse a cabo.

El contexto social y político estaba en tensión por el paro magisterial en la entidad, como protesta frente a la reforma educativa que modificó la relación laboral del magisterio con el gobierno. Los discursos en favor y en contra confluían sistemáticamente hacia el tema de la evaluación de desempeño docente. En este marco, el grupo de estudiantes de maestría se acercó a la evaluación con estándares de desempeńo, y al aplicarla encontró un modelo que permitió generar información para la mejora de su práctica de manera directa y en colaboración con sus colegas de trabajo.

Ante las posturas encontradas respecto a la defensa o abrogación de la ley sobre la actual evaluación de desempeño docente, la evaluación con estándares es una alternativa cercana a la práctica de los profesionales de la educación. La experiencia sistematizada en este documento muestra un camino por el que se puede transitar hacia una discusión sobre los atributos y las necesidades reales del personal docente en vistas de su mejora profesional y, en consecuencia, de la formación de niñas, niños y adolescentes.

Los hallazgos más relevantes de este ejercicio muestran que es necesario tomar con seriedad que diversos actores escolares se involucren en la evaluación. Esto es algo que ya la SEP y el Instituto Nacional para la Evaluación de la Educación han incorporado en la evaluación del desempeño, lo cual, en fechas recientes, modificaron, y ahora prevé fortalecer la autoevaluación mediante la identificación de fortalezas y debilidades (INEE, 25 de agosto de 2016). Si bien, la participación de los actores representa ventajas por la triangulación de información que se puede hacer, aún está pendiente que el proceso se oriente a la mejora, que genere reflexión y no solo rendición de cuentas. Esto implica que los actores escolares convocados pasen de ser evaluadores (copartícipes 
del proceso), a corresponsables de los resultados. Esta comprensión de la evaluación del desempeño, aún está por construirse.

Adicionalmente, la experiencia sistematizada en este artículo arroja información respecto a que los docentes que han sido capacitados para autoevaluarse, y que cuentan con elementos claros respecto a lo que han de observar en sus clases, pueden llegar a ser incluso más críticos con su propia práctica, que sus pares o sus autoridades. Esto se observa en la diferencia de valoraciones que emitieron autoevaluador, coevaluador y heteroevaluador en torno a los mismos referentes. La presencia sistemática de resultados como este confirmaría que es un acierto el incluir la autoevaluación, no sólo por tratarse de un mecanismo de obtención de información de primera mano, sino también por la oportunidad de formación que representa para quienes la llevan a cabo.

El modelo de estándares desata un proceso de aprendizaje y formación recíproca entre las y los evaluadores, porque los criterios para evaluar están depositados en los instrumentos de registro y valoración, y no en la experiencia o falta de esta por parte de quien está siendo un observador. En la medida en que se sigue haciendo uso del modelo, los evaluadores adquieren conocimientos y habilidades que derivan en procesos de evaluación más reflexivos y desarrollados en ambientes de confianza y solidaridad. De esta manera todos aprenden, evitan prejuicios, y pueden diseñar estrategias y modos de gestionar el aprendizaje en el aula, distintos de los que antes cada uno realizaba por su cuenta.

Por otra parte, la cercanía en las valoraciones de los tres evaluadores respecto a las mismas categorías (aun cuando, como hemos señalado, hay variación en cada referente) indica que existe una coincidencia respecto a la ubicación de las fortalezas y debilidades de la práctica. En este punto es importante no quedarse con el dato numérico del valor, sino profundizar en el diálogo sobre las justificaciones de sus valoraciones en vista de un trayecto de mejora de la práctica docente. Cuál es el foco de atención de cada evaluador, y cuáles los argumentos que sustentan su valoración son temas para futuras investigaciones.

Los trayectos de mejora reúnen también información altamente relevante del proceso. En esta experiencia se encontró que las temáticas de las acciones contenidas en los trayectos se relacio- 
nan con los referentes, lo cual es un indicador de que estos coadyuvan a ampliar los radios de acción que docentes y evaluadores consideran para transformar su práctica.

En los resultados esperados del trayecto de mejora, los docentes hacen alusión a sus expectativas, y es interesante observar que en ello sitúan tanto las transformaciones de su quehacer en el ámbito individual, como en el de su participación colectiva. Una línea de investigación pendiente sería indagar qué tanto los resultados de los trayectos de mejora empatan con lo que se pretende en el sistema educativo, y en particular, con la reforma.

Asimismo, está pendiente para el grupo evaluar cómo se ha de poner en juego el trayecto de mejora, particularmente, al darse cuenta de que se pueden recabar otras evidencias del quehacer docente, diferentes de la planeación. La construcción de una mirada más comprensiva al aula es un proceso lento y de largo plazo, donde los actores escolares habrán de reconocer la diferencia entre evaluar el decir y evaluar el hacer.

Cabe mencionar que la elaboración de seguimientos representó un reto para este grupo, pues hay muchas semejanzas en cuanto a las temáticas y recursos mencionadas en los trayectos. El seguimiento es una herramienta para acompañar, no es el trayecto en sí mismo, no se trata de duplicar funciones, sino de hacer explícito cómo habrá de llevarse a cabo el acompañamiento al docente.

El ejercicio comparativo de relación entre valoraciones, trayectos y seguimientos permite observar que las valoraciones más bajas no siempre son los temas que se atienden en el trayecto; esto abre una ventana de investigación muy interesante respecto a cómo los evaluadores toman las decisiones de qué y cuándo modificar. Entender qué ocurre de la valoración a la elaboración del trayecto resulta clave para saber qué es lo que más pesa a la hora de tomar estas decisiones.

Sobre este punto valen dos hallazgos: el primero, que en todos los casos, sistemáticamente, se están quedando sin atender los referentes más bajos de la categoría evaluación, lo que puede estar evidenciando la necesidad de desarrollar estrategias para el uso de los docentes en el aula. Una hipótesis es que el énfasis en las evaluaciones externas podría estar conduciendo a que la evaluación 
en todas sus posibilidades en el aula, no sea apreciada por ellos como un ámbito de acción.

Es muy interesante el hecho de que los trayectos de mejora y seguimientos puedan ser abordajes en colectivo, para la atención de necesidades escolares y no solo individuales; sin embargo, deberá tomarse en cuenta, como ocurre en la experiencia de este seminario, que el avance en colectivo no puede excluir la atención inmediata a las necesidades individuales evidenciadas por los referentes. Una conciliación de los dos ámbitos es imprescindible para que el ejercicio continúe siendo pertinente a la necesidad del aula.

Una de las cualidades más importantes de este ejercicio es que revela que su continuidad está en función del compromiso que hacen diferentes actores cuando se comprometen como corresponsables de la mejora de la práctica.

\section{REFERENCIAS BIBLIOGRÁFICAS}

Animal Político. "Maestros bloquean el D. F.", en Animal Político, 22 de agosto 2013. Disponible en http://www. animalpolitico.com/2013/08/maestros-asedian-tambiensede-alterna-la-ciudad-es-un-caos/

Centro de Estudios Educativos. Referentes para la mejora de la educación básica. Informe del proceso de legitimación y validación, 470, México, CEE, 2008. Disponible en http:// referenteseducativos.net/images/pdf-fundamentos/estandarescurriculares.pdf

Centro de Estudios Educativos. Estándares de desempeño docente en el aula. Cuadernillo 1. Manual para la aplicación del instrumento, Ciudad de México, CEE, 2010a. Disponible en http://cee.edu.mx/referentes/

Centro de Estudios Educativos. Estándares de Desempeño Docente en el Aula. Cuadernillo 2. Formato de Autorregistro e Instrumento de Estándares de Desempeño Docente en el Aula, Ciudad de México, CEE, 2010b. Disponible en http://cee. edu. $\mathrm{mx} /$ referentes/ 
Garduño, R. y Méndez E. "Sesiona a salto de mata la Cámara de Diputados, dice el PT en la sede alterna”, en periódico La Jornada, 22 de agosto de 2013. Disponible en http:// www.jornada.unam.mx/2013/08/22/politica/010n1 pol

Herrera, C. "Sin la presencia de Gordillo fue promulgada la reforma educativa”, en periódico La Jornada, 26 de febrero de 2013. Disponible en http://www.jornada.unam. $\mathrm{mx} / 2013 / 02 / 26 /$ politica/005n 1 pol

Instituto Nacional para la Evaluación de la Educación. "Replantea el INEE el modelo de evaluación del desempeño profesional docente para 2017", Ciudad de México, INEE, 2016. Disponible en http://www.inee.edu.mx/images/ stories/2016/boletines/Boletin47.pdf

Román, J. "Reafirmar la rectoría del Estado en materia educativa, plantea Peña", en periódico La Jornada, 11 de diciembre 2012. Dsponible en http://www.jornada.unam. $\mathrm{mx} / 2012 / 12 / 11 /$ politica/002n1pol

Subsecretaría de Educación Básica. Documento base. Estándares de Desempeño Docente en el aula para la educción básica en México, Ciudad de México, Secretaría de Educación Pública, 2010. Disponible en http://cee.edu.mx/referentes/2-2.pdf

Ureste, Manuel. "Diputados discuten en sede alterna la Ley General de Educación", en Animal Político, agosto 21 de 2013, 22:54 hrs. Disponible en http://www.animalpolitico.com/2013/08/diputados-inician-sesion-extraordinaria-en-sede-alterna-con-trifulca/. Fecha de consulta, 21 de agosto de 2013. 
